\title{
R.E.N.A.L. Nephrometry Score Profile in Kidney Cancer Patients at Cipto Mangunkusumo Hospital (CMH)
}

\author{
Rinto Hariwibowo, Agus Rizal AH Hamid, Chaidir Arif Mochtar
}

Department of Urology, Faculty of Medicine Universitas Indonesia, Dr. Cipto Mangunkusumo Hospital, Jakarta, Indonesia

\section{ARTICLE INFO}

Received : 12 June 2019

Reviewed : 21 June 2019

Accepted : 01 August 2019

\section{Keywords:}

kidney cancer, nephrectomy, R.E.N.A.L nephrometry

\author{
*Corresponding author: \\ Chaidir Arif Mochtar \\ Department of Urology, \\ Faculty of Medicine Universitas \\ Indonesia, Dr. Cipto Mangunkusumo \\ Hospital, Jakarta, Indonesia. \\ chamochtar@gmail.com
}

\begin{abstract}
Background: The variation of sizes, shapes, and location of kidney cancer complicates the choices of surgical treatment. To determine which technique to use, R.E.N.A.L. nephrometry scoring (NS) systems were established. This study was conducted to evaluate R.E.N.A.L.-NS profile in kidney cancer patients at Cipto Mangunkusumo Hospital (CMH).
\end{abstract}

\begin{abstract}
Methods: The data were collected retrospectively from patients that underwent both open and laparoscopic radical (RN) and partial nephrectomy (PN) procedures from 2014-2017. R.E.N.A.L.-NS was calculated based on (R)adius, (E)xophytic/Endophytic properties, (N)earness to the collecting system, (A)nterior or Posterior position of the tumor, and (L)ocation of the tumor. Data were categorized into three complexities: low (4-6 points), medium (7-9 points), and high (10-12 points). Subjects were then divided based on the given procedure. Profile of R.E.N.A.L.-NS was shown based on each procedure.
\end{abstract}

Results: In this study, 63 patients were included. Fifty two patients underwent RN and 11 underwent PN. In low complexity tumors, all patients received PN. In medium complexity tumors, 22 (78.5\%) patients received RN and $6(21.5 \%)$ received PN. All high complexity tumors received RN. Mean renal score in all patients were $9.03( \pm 1.72)$, RN $9.59( \pm 1.11)$, PN $6.36( \pm 1.6)$. Higher $(R),(N)$, and $(L)$ scores mean a higher prevalence of RN.

Conclusions: Higher complexity tumors were more likely to be treated with RN. Furthermore, $(\mathrm{R})$, (N), and (L) score can be useful to determine RN or PN as the treatment of choice. This study could be used as a reference to another study regarding R.E.N.A.L.-NS in Indonesia.

\section{INTRODUCTION}

Kidney cancer is one of the most common forms of cancer in Indonesia, ranked third of all urogenital tumors with an incidence of 2,093 per 100,000 population and was estimated to have 5 -years prevalence of 4,444 per 100,000 population in 2012 [1-6]. Renal cell carcinoma (RCC) comprises approximately $90 \%$ of all malignant renal tumors [1]. Surgical treatment still remains as the standard care for patients with localized renal malignancies $[1,3,7-10]$. The variation of sizes, shapes, and location of the tumor complicates the choice of surgical treatment for patients with renal tumors which give challenges for the urologist to determine which technique to be used for excising localized renal tumors $[7,11]$.

In the last three decades, the option for tumor excision is not only limited to radical nephrectomy (RN), nephron-sparing surgery or partial nephrectomy (PN) has emerged as one of the oncological equivalent alternative to $\mathrm{RN}$ in most cases of localized renal tumors $[1,8,9]$. More recent technology such as laparoscopy also emerged and thus further complicating the treatment of choices for renal tumors [1]. This could result in treatment choices bias subject to the surgeon's training patterns, surgeon's comfort levels, and their individual experiences $[1,12,13]$.

To help to objectify renal masses and to help urologist to determine which technique to be used, several scoring systems were established [13]. These several scoring systems are nowadays used as treatment decision-making for urologist, worldwide $[1,12,13]$. R.E.N.A.L.-NS was the first known scoring system, published by Kutikov and Uzzo in 2009, and have been used worldwide to determine treatment options for RCC [1]. This R.E.N.A.L.-NS is still not widely used in Indonesia. This study was conducted to evaluate the R.E.N.A.L.-NS profile of RCC patients in Cipto Mangunkusumo Hospital (CMH), as the foundation for other further studies in Indonesia. 


\section{METHODS}

\section{Participant}

The subjects of this study were renal tumor patients in $\mathrm{CMH}$ from 2014-2017 that underwent RN and PN procedure in $\mathrm{CMH}$, including both open and laparoscopic procedures. The data for this study were collected retrospectively from our medical record databases, including computed tomography scan (CT-scan) and patient's tumor and perioperative profiles. Patients with insufficient CT-scan data were excluded from this study. R.E.N.A.L.-NS was calculated based on the following anatomical features of renal tumor: (R)adius of renal tumors (maximal diameter in centimeters), (E)xophytic/ Endophytic properties, (N)earness of the tumor to the collecting system or sinus (in millimeters), (A)nterior or Posterior position of tumor (not shown as point, but with descriptor based on the exact location of tumor), and (L)ocation of tumor relative to the polar line (Table 1.) $[1,14]$. This study has been approved by the ethics committee in CMH, 284/UN2.F1/ETIK/2016.

\section{Data Analysis}

Based on the scoring, the tumors were categorized into three complexity classes, which are low complexity (4-6 points), medium complexity (7-9 points), and high complexity (10-12 points). Demographic data and general profile of R.E.N.A.L.-NS were collected including age, gender, tumor radius, treatment approach, mean renal score, anterior and posterior location, and tumor complexity. Subjects were then divided based on the procedure given (radical nephrectomy or partial nephrectomy) and the profile of the (R), (E), (N), and (L) scores of the R.E.N.A.L.-NS. The subgroup analysis between tumor complexity with treatment received (radical or partial nephrectomy) and with treatment approach (open or laparoscopic) was performed using Chi-square test. All statistical analysis was analyzed with SPSS 16.0.

\section{RESULTS}

There were 63 patients included in this study, of which 52 patients underwent radical nephrectomy (RN) and 11 patients underwent partial nephrectomy (PN). The characteristics of the patients are shown in Table 2. The age of patients ranges from 25 to 76 years old, with a median of 53 years old. The majority of the patients were male $(68 \%)$, compared to female (32\%). There was a relatively equal trend between the open and laparoscopic approach in radical nephrectomy patients. In partial nephrectomy patients, the laparoscopic approach was more common compared to the open approach.
The average RENAL score of all patients was around nine, which falls into the medium complexity category. Radical nephrectomy patients had higher mean RENAL score compared to partial nephrectomy patients. Most of the patients were of high complexity and medium complexity tumors. In radical nephrectomy group, we did not have low complexity tumors, while more than half of the patients were of high complexity. In partial nephrectomy group, low and medium complexity tumors were almost equally prevalent, with none of the patients was of high complexity. We also had three laparoscopic patients with medium complexity tumors who had an intraoperative conversion from partial nephrectomy to radical nephrectomy, due to the inadequate surgical margin of the excised tumor. For tumor location, we found that half of all tumors were located posteriorly (52.4\%). A similar percentage was found within radical nephrectomy group and partial nephrectomy group.

Table 3 showed the R.E.N.A.L.-NS profile for each procedure: radical nephrectomy $(\mathrm{RN})$ and partial nephrectomy $(P N)$, especially the $(R),(E),(N)$, and $(L)$ score because the $(A)$ score had been described in Table 2 .

For the radius $(\mathrm{R})$ score of 3 , radical nephrectomy was more prevalent (94\%) than partial nephrectomy $(6 \%)$. For $(R)$ score of 2 , more than half of the patients received $P N$. All patients with $(R)$ score of 1 received partial nephrectomy. All patients with exophytic/ endophytic (E) score of 3 received radical nephrectomy. In (E) score of 2 and 1, lower (E) score showed higher percentage of partial nephrectomy, from $15.4 \%$ partial nephrectomy rates in (E) score of 2 to $31.2 \%$ in (E) score of 1 . For the nearness $(\mathrm{N})$ score, there was a higher percentage of radical nephrectomy in higher $(\mathrm{N})$ scores (96\% radical nephrectomy in (N) score of 3 ) and higher percentage of partial nephrectomy in lower (N) scores (100\% partial nephrectomy in $(N)$ score of 1$)$. For the location of tumor relative to polar lines (L) score, all patients with (L) score of 3 received radical nephrectomy and there was a higher percentage of partial nephrectomy in the lower (L) score compared to higher (L) score.

Table 4 showed an analysis of tumor complexity subgroup and the treatment received by patients. All low complexity tumors in our study received partial nephrectomy. Twenty-two patients (75.8\%) in medium complexity group received radical nephrectomy. All high complexity tumors were treated with radical nephrectomy. These results showed statistical significance based on the Chi-square test $(p<0.01)$. Further subgroup analysis of open versus laparoscopic approach in each radical and partial nephrectomy group showed no statistical significance. An interesting finding was that laparoscopy was performed more frequently than open surgery almost in all subgroups, except in high complexity tumors where open surgery still more prevalent than laparoscopic. 
Table 1. R.E.N.A.L. Nephrometry Scoring Components

\begin{tabular}{|c|c|c|c|}
\hline Parameters & $1 \mathrm{pt}$ & 2 pts & 3 pts \\
\hline (R)adius in $\mathrm{cm}$ & $\leq 4$ & $>4$ but $<7$ & $\geq 7$ \\
\hline (E)xophytic/endophytic properties & $\geq 50 \%$ & $<50 \%$ & Tumor entirely endophytic \\
\hline$(\mathrm{N})$ earness of the tumor to collecting system in $\mathrm{mm}$ & $\geq 7$ & $>4$ but $<7$ & $\leq 4$ \\
\hline (A)nterior/Posterior & \multicolumn{3}{|c|}{ No points given. Mass assigned a descriptor $a, p$, or $x$} \\
\hline $\begin{array}{l}\text { (L)ocation relative to the polar lines } \\
\text { Suffix " } h \text { " given if tumor touches } \\
\text { the main renal artery or vein }\end{array}$ & $\begin{array}{l}\text { Entirely above } \\
\text { the upper polar } \\
\text { line or below } \\
\text { the lower polar } \\
\text { line }\end{array}$ & $\begin{array}{l}\text { Lesion crosses } \\
\text { the polar line }\end{array}$ & $\begin{array}{l}>50 \% \text { of the mass is across } \\
\text { the polar line, or mass crosses } \\
\text { axial renal midline or mass is } \\
\text { entirely between the polar lines }\end{array}$ \\
\hline
\end{tabular}

Table 2. Demographic data of patients that underwent RN and PN in CMH from 2014-2017

\begin{tabular}{|c|c|c|c|}
\hline Characteristics & $\begin{array}{l}\text { All Patients } \\
(n=63)\end{array}$ & $\begin{array}{l}\text { Radical Nephrectomy } \\
\qquad(\mathrm{n}=52)\end{array}$ & $\begin{array}{l}\text { Partial Nephrectomy } \\
\qquad(\mathrm{n}=11)\end{array}$ \\
\hline Age, years (median) & $53(25-76)$ & $51(25-76)$ & $61(46-71)$ \\
\hline \multicolumn{4}{|l|}{ Gender } \\
\hline Male & 43 & 36 & 7 \\
\hline Female & 20 & 16 & 4 \\
\hline \multicolumn{4}{|l|}{ Tumor } \\
\hline Radius/Diameter in $\mathrm{mm}( \pm \mathrm{SD})$ & $114( \pm 57)$ & $124( \pm 55)$ & $64.5( \pm 33.5)$ \\
\hline \multicolumn{4}{|l|}{ Approach } \\
\hline Open & 32 & 28 & 4 \\
\hline Laparoscopy & 31 & 24 & 7 \\
\hline Mean Renal Score & $9.03( \pm 1.72)$ & $9.59( \pm 1.11)$ & $6.36( \pm 1.6)$ \\
\hline Anterior Location & $9(14.3 \%)$ & $8(11.5 \%)$ & $3(27.2 \%)$ \\
\hline Posterior Location & $33(52.4 \%)$ & $28(53.9 \%)$ & $6(54.6 \%)$ \\
\hline X Location & $21(33.3 \%)$ & $18(34.6 \%)$ & $2(18.2 \%)$ \\
\hline Low Complexity & $5(7.9 \%)$ & $0(0 \%)$ & $5(45.5 \%)$ \\
\hline Medium Complexity & $28(44.5 \%)$ & $22(42.3 \%)$ & $6(54.5 \%)$ \\
\hline High Complexity & $30(47.6 \%)$ & $30(57.7 \%)$ & $0(0 \%)$ \\
\hline
\end{tabular}

Table 3. R.E.N.A.L. Nephrometry Score Profile in all Patient's Group

\begin{tabular}{|c|c|c|}
\hline $\begin{array}{c}\text { Nephrometry Score } \\
\text { Parameters }\end{array}$ & $\begin{array}{c}\text { Radical } \\
\text { Nephrectomy } \\
(n=52)\end{array}$ & $\begin{array}{c}\text { Partial } \\
\text { Nephrectomy } \\
(n=11)\end{array}$ \\
\hline Radius & $\begin{array}{l}3=48(94 \%) \\
2=4(44.4 \%) \\
1=0(0 \%)\end{array}$ & $\begin{array}{l}3=3(6 \%) \\
2=5(55.6 \%) \\
1=3(100 \%)\end{array}$ \\
\hline Exophytic/ Endophytic & $\begin{array}{l}3=8(100 \%) \\
2=33(84.6 \%) \\
1=11(68.8 \%)\end{array}$ & $\begin{array}{l}3=0(0 \%) \\
2=6(15.4 \%) \\
1=5(31.2 \%)\end{array}$ \\
\hline $\begin{array}{l}\text { Nearness of tumor } \\
\text { to renal sinus }\end{array}$ & $\begin{array}{l}3=25(96 \%) \\
2=27(84.4 \%) \\
1=0(0 \%)\end{array}$ & $\begin{array}{l}3=1(4 \%) \\
2=5(15.6 \%) \\
1=5(100 \%)\end{array}$ \\
\hline $\begin{array}{l}\text { Location relative to } \\
\text { polar lines }\end{array}$ & $\begin{array}{l}3=22(100 \%) \\
2=23(88 \%) \\
1=8(50 \%)\end{array}$ & $\begin{array}{l}3=0(0 \%) \\
2=3(12 \%) \\
1=8(50 \%)\end{array}$ \\
\hline
\end{tabular}

Table 4. Patient's tumor complexity subgroup analysis

\begin{tabular}{ccccc}
\hline & \multicolumn{4}{c}{ Complexity } \\
\cline { 2 - 5 } & $\begin{array}{c}\text { Low } \\
\text { Complexity } \\
(\mathrm{n}=5)\end{array}$ & $\begin{array}{c}\text { Medium } \\
\text { Complexity } \\
(\mathrm{n}=29)\end{array}$ & $\begin{array}{c}\text { High } \\
\text { Complexity } \\
(\mathrm{n}=29)\end{array}$ & $\mathrm{p}$ \\
\hline Treatment & & & & \\
RN (radical) & $0(0 \%)$ & $22(78,5 \%)$ & $30(100 \%)$ & $<0.01$ \\
PN (partial) & $5(100 \%)$ & $6(21.5 \%)$ & $0(0 \%)$ & \\
\hline RN (radical) & & & & \\
Open & $0(0 \%)$ & $9(40.9 \%)$ & $19(63.3 \%)$ & \\
Laparoscopy & $0(0 \%)$ & $13(59.1 \%) ;$ & $11(36.7 \%)$ & $>0.05$ \\
\hline PN (partial) & & & & \\
Open & $2(40 \%)$ & $2(33.3 \%)$ & $0(0 \%)$ & $>0.05$ \\
Laparoscopy & $3(60 \%)$ & $4(66.7 \%)$ & $0(0 \%)$ & \\
\hline
\end{tabular}




\section{DISCUSSION}

R.E.N.A.L.-NS was developed by Kutikov and Uzzo in 2009 with the hope to standardize the assessment of renal tumors' anatomical features. R.E.N.A.L.-NS itself consists of (R)adius subscale (tumor size at maximal diameter), (E)xophytic/endophytic subscales that explains the exo/endophytic properties of tumor, (N) subscales that is defined as nearness of tumor to collecting system or sinus, (A)nterior/posterior subscale with a as descriptor for anterior location, $p$ as descriptor for posterior location, and $\mathrm{x}$ as descriptor for neither anterior nor posterior (could not be defined), and ( $L$ ) ocation of tumor relative to the polar line. Suffix " $h$ " or hilar is assigned for a tumor that is close or touches the main renal artery or vein [1-7,11,14-17]. R.E.N.A.L.NS could be scored based on CT or MRI findings of patients at diagnosis [1]. Because R.E.N.A.L.-NS was developed to evaluate the complexity of renal tumors quantitatively, many studies are now performed to confirm the usefulness of R.E.N.A.L.-NS, whether for decision-making (treatment choices) as performed by Canter et al. [12], for predicting outcomes as performed by Kopp et al. [15] and Nagahara et al. [14], and many more. Several other scoring systems have also been described, such as the Preoperative Aspects and Dimensions Used for an Anatomical Classification System (PADUA score) and the Centrality index (C-index score). Okhunov et al. [13] stated that R.E.N.A.L.-NS was more appealing compared to the C-Index score, due to its simplicity. Borgmann et al. [18] also found R.E.N.A.L.-NS to have good correlations with perioperative outcomes in nephron-sparing surgery. In Indonesia, there is still no study that try to correlate R.E.N.A.L.-NS with all outcomes and procedures. Our study tried to breakdown the profile of R.E.N.A.L.-NS in CMH from 2014-2017 to see how R.E.N.A.L.-NS correlates with the tumor and with procedures that are done in $\mathrm{CMH}$.

From all 63 patients that were included, tumors were treated with radical and partial nephrectomy, with both open and laparoscopic technique. The number of partial nephrectomy is still not many in our center because most of the patients that come to our center are already at a later stage, considering that our center is the national referral center.

In our present study, from 52 patients that were treated with radical nephrectomy, 29 patients were categorized as high complexity tumor ( $100 \%$ of total) and 23 were categorized as moderate complexity tumor ( $80 \%$ in total), and none were categorized as low complexity tumor. This data correlates well with the study conducted by Canter et al. [14] and Wong et al. [6] which showed that patients treated with radical nephrectomy were of moderate and high complexity; none were of low complexity. It could be highlighted that in patients that underwent partial nephrectomy, there were 5 patients that were categorized as low complexity tumors, contributing to $100 \%$ of total patients. It was different from the radical nephrectomy group where there were no patients categorized as low complexity tumor. This profile correlates well with Canter et al. [14] study and Wong et al. [6] study. From both of those studies, it was shown that patients with low complexity tumor and some of the moderate complexity tumors were being treated with nephronsparing surgery/partial nephrectomy $[6,14]$. Overall, with higher complexity, it is more likely for the tumor to be treated with radical nephrectomy [14].

Our study showed that in cases with higher (R), $(\mathrm{N})$, and (L) scores, radical nephrectomy was much more prevalent than partial nephrectomy. This result concordance with the study conducted by Canter et al [14]. In their study, patients with high (R) and (L) score will most likely undergo radical nephrectomy. For the (E) and (N) scores in their study, there was no increasing percentage of radical nephrectomy in higher (E) score (only 43\% radical nephrectomy in (E) score of 3 compared to $35 \%$ in (E) score of 1 ); but there was an increasing percentage of radical nephrectomy for higher $(N)$ score $143.2 \%$ radical nephrectomy in $(\mathrm{N})$ score of 3 compared to only $11 \%$ in (N) score of 1 ). They also analyzed the scores of partial nephrectomy patients treated with minimally invasive surgery (183 patients) and open surgery (224 patients), and found higher (R), (E), and (N) scores to be correlated with open surgery compared to the laparoscopic approach.

To perform such subgroup analysis in our center, more partial nephrectomy cases are require which were still limited in our center. Our study also had limited samples of 63 patients, and because this study was mainly aimed to describe the demographics of kidney cancer complexity levels and treatments, minimal statistical analysis was performed. We propose that in the future, larger multicentric studies may be initiated to help defining kidney cancers better.

\section{CONCLUSIONS}

From our data, it is shown that the higher the complexity of tumor, the more likely it is to be treated with radical nephrectomy. Furthermore, $(R),(N)$, and (L) score can be useful to determine radical or partial nephrectomy as a treatment of choice for renal tumors. This present study could be used as a reference to other study regarding R.E.N.A.L.-NS in Indonesia. 


\section{DECLARATIONS}

\section{Competing of Interest}

The authors affirm no conflict of interest in this study.

\section{Acknowledgement}

The authors would like to express our gratitude to everyone that contributed in the making of this paper.

\section{REFERENCES}

1. Kutikov A, Uzzo RG. The R.E.N.A.L. Nephrometry score: A comprehensive standardized system for quantitating renal tumor size, location, and depth. J Urol. 2009;182: 844-53.

2. Monn MF, Gellhaus PT, Masterson TA, Patel AA, Tann $M$, Cregar DM, et al. R.E.N.A.L. Nephrometry scoring: How well correlated are urologist, radiologist, and collaborator scores?. J Endourol. 2014;28: 1006-10.

3. Zhou HJ, Yan Y, Zhang JZ, Liang LR, Guo SB. Role of R.E.N.A.L. nephrometry score in laparoscopic partial nephrectomy. Chin Med J (Engl). 2017;130: 2170-5.

4. Mottrie A, Gandaglia G. Do we need a novel nephrometry scoring system in partial nephrectomy?. Eur Urol. 2016;69: 80-1.

5. Reddy UD, Pillai R, Parker RA, Weston J, Burgess NA, Ho ETS, et al. Prediction of complications after partial nephrectomy by RENAL nephrometry score. Ann R Coll Surg Engl. 2014;96: 475-9.

6. Wong M, Cho K, Ho K, Lai CT, Man CM, Yiu MK. How can the R.E.N.A.L. nephrometry scoring system aid management of a solid renal mass?. Hong Kong Med J. 2013;20: 37-44.

7. Shaaban MS, Youssif TMA, Mostafa A, Hegazy HE, Atta MA. Role of RENAL nephrometry scoring system in planning surgical intervention in patients with localized renal masses. Egypt J Radiol Nucl Med. 2015;46: 1175-81.

8. Umbas R, Hardjowijoto S, Safriadi F, Mochtar CA, Djatisoesanto $W$, Soedarsono $M A$, et al. Guidelines on renal malignant tumour. Indones Urol Assoc. 2012;1-7.
9. Ljungberg B, Bensalah K, Vice-chair AB, Bex A, Giles $\mathrm{RH}$, Hora $M$, et al. EAU Guidelines on renal cell carcinoma. Eur Assoc Urol. 2017;1-62.

10. Carroll RN, BSN, MBA CM. Kidney cancer. NCCN Clin Pract Guidel Oncol 2018;3: 4-43.

11. Parsons RB, Canter D, Kutikov A, Uzzo RG. RENAL nephrometry scoring system: The radiologist's perspective. Am J Roentgenol. 2012;199:355-9.

12. Canter D, Kutikov A, Manley B, Egleston B, Simhan $J$, Smaldone $M$, et al. Utility of the R.E.N.A.L. nephrometry scoring system in objectifying treatment decision-making of the enhancing renal mass. Urology. 2011;78: 1089-94.

13. Okhunov Z, Rais-Bahrami S, George AK, Waingankar $\mathrm{N}$, Duty $\mathrm{B}$, Montag $\mathrm{S}$, et al. The comparison of three renal tumor scoring systems: C-Index, P.A.D.U.A., and R.E.N.A.L. nephrometry scores. J Endourol. 2011;25: 1921-24.

14. Nagahara A, Uemura M, Kawashima A, Ujike T, Fujita $K$, Miyagawa $Y$, et al. R.E.N.A.L. nephrometry score predicts postoperative recurrence of localized renal cell carcinoma treated by radical nephrectomy. Int J Clin Oncol. 2016;21: 367-72.

15. Kopp RP, Mehrazin R, Palazzi KL, Liss MA, Jabaji R, Mirheydar MS, et al. Survival outcomes after radical and partial nephrectomy for clinical T2 renal tumours categorised by R.E.N.A.L. nephrometry score. BJU Int. 2014;114: 708-18.

16. WHO. GLOBOCAN: Estimated cancer incidence, mortality, and prevalence worldwide in 2012. c2012 - [cited 2018 February 1]. Available from: http:// globocan.iarc.fr/Pages/fact_sheets_population.aspx

17. Lin TP, Kao YM, Chen M, Sun FJ, Lin WR. Functional outcome prediction after partial nephrectomy using R.E.N.A.L. nephrometry, PADUA classification, and Centrality index score. Urol Sci. 2017;28: 10-3.

18. Borgmann $H$, Reiss AK, Kurosch M, Filmann N, Frees $S$, Mager R, et al. R.E.N.A.L. score outperforms PADUA score, C-Index and DAP score for outcome prediction of nephron sparing surgery in a selected cohort. J Urol. 2016;196: 664-71. 